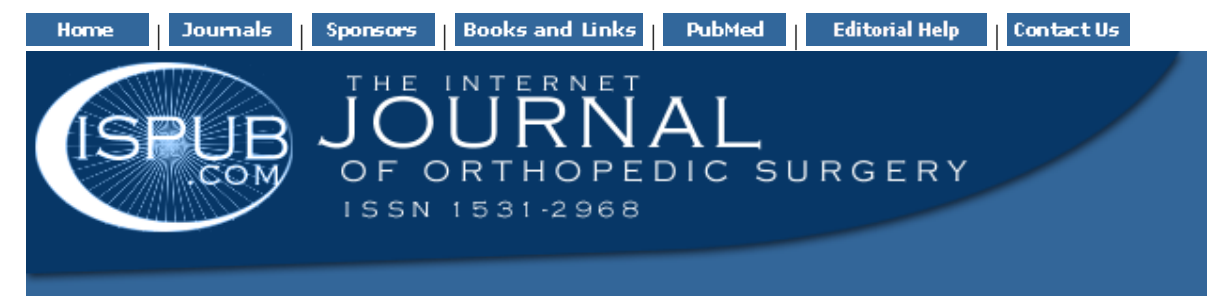

Advertisement

(2)

Web www.ispub.com Google Search

AWARDS
Advertisement

The Internet Journal of Orthopedic Surgery ISSN: 1531-2968

Home | Current Issue | Archives | Instructions for Authors | Disclaimer | Printable Version

\section{Percutaneous Trans Ilio-Sacral Biopsy Of The Spine In Sacral} Tuberculosis

Hilgaard Frans Visser, MBChB

Department of Orthopaedic Surgery

Kalafong hospital

University of Pretoria

South Africa

Nkosana Silas Motsitsi, M.MED. F.C.S.

Department of Orthopaedic Surgery

Kalafong hospital

University of Pretoria

South Africa

\section{Citation:}

Hilgaard Frans Visser, Nkosana Silas Motsitsi: Percutaneous Trans llio-Sacral Biopsy Of The Spine In Sacral Tuberculosis. The Internet Journal of Orthopedic Surgery. 2008. Volume 8 Number 1.

Keywords: sacral tuberculosis, percutaneous technique, ilio-sacral approach

\section{Table of Contents}

\section{Abstract}

Background

Case presentation

Discussion

Conclusion

References

\begin{abstract}
Spinal tuberculosis is the most common form of skeletal tuberculosis. Tuberculous involvement of the lower spine is rare. Sacral tuberculosis is very rare; most reported cases in the literature are case reports. Spinal tuberculosis may present atypically: neural arch involvement, skip lesions, sacral involvement, or as extradural tuberculosis. Diagnostic bone biopsy is an integral part of patient work-up. Patients may be infected by a variety of micro organisms, especially patients who are HIV positive or those who have AIDS.

The gold standard technique for biopsy of the sacrum is CT-guided percutaneous biopsy. The technique has a very low complication rate in experienced hands. We describe a simple, safe and fast percutaneous biopsy technique we used to obtain biopsy of the upper sacrum. The details are stated in this report.
\end{abstract}

\section{Background}

Tuberculous infection of the musculo-skeletal system constitutes about $1 \%$ of all tuberculous infections. Spinal tuberculosis constitutes about one quarter to two-thirds of musculo-skeletal tuberculosis[ $\left.{ }^{1}\right]$. 
The distribution of spinal tuberculosis(TB) varies widely according to the literature: the commonest affected site is the thoraco-lumbar region, followed by the thoracic and then the lumbar area. The lumbo-sacral area is affected in less than $5 \%$ of cases $\left[{ }^{2},{ }^{3}\right]$. Isolated sacral TB is extremely rare[ $\left.{ }^{4}\right]$.

The high prevalence of HIV and AIDS has caused an increase in the incidence of not only TB in general, but also an increase in spinal tuberculosis, especially in developing countries. HIV positive patients can be infected by a variety of organisms; spinal biopsy becomes an important part of patients' diagnostic work-up.

We present a case of a young HIV positive male patient who had sacral tuberculosis. We describe a technique called percutaneous trans ilio-sacral biopsy. To the best of our knowledge, this technique has not been described in the English literature.

We detail the presentation, physical examination, and the percutaneous trans ilio-sacral biopsy technique.

\section{Case presentation}

A 31 year-old HIV positive male patient was referred to our spinal clinic because of chronic progressive lower backache. The pain started spontaneously about one year before presentation. He has been treated conservatively at the local clinic where he received antiretroviral therapy. He had no complaint of bladder or rectal sphincter dysfunctions. He was complaining of pain in the lower limbs and it was getting progressively worse. There were no complaints relating to neurology. Family history was unremarkable.

On physical examination, he was a well and healthy-looking young man. There were no findings generally. Local findings of the lower back showed deformity in the S1/S2 area, localized tenderness, no local signs of inflammation or abscess formation, and he had tenderness on both sacro-iliac joints. Neurological examinations showed; decrease sensation to light touch and pinprick bilaterally in the S2- S4 dermatomes, normal motor power bilaterally, and normal reflexes. He had normal rectal sphincter tone and function. There were no any other significant findings. A clinical diagnosis of spinal tuberculosis was made.

Plain radiographs of the spine were requested. The $\mathrm{X}$-rays showed destruction of the upper sacral spine( S1/S2) with possible involvement of the sacro-iliac joints. Computed tomography scan ( CAT-SCAN) was requested. CAT SCAN with contrast was done: it showed extensive S1 and S2 destruction with disc space narrowing, There was an extensive pre-vertebral soft tissue mass measuring $7.0 \mathrm{~cm} \times 8.0 \mathrm{~cm}$ stretching from the inferior border of L5/S1 disc space proximally to the superior border of S4 inferiorly. The mass extended into the vertebral canal thus displacing the neural tissue posteriorly. The mass was well-encapsulated. There were areas of calcifications within the mass (Figure 1).

Figure 1: CT scan showing sagittal view of the sacrum. There is a huge cold abscess in front of the sacral vertebrae. It also involves the spinal canal. It is well encapsulated. There are multiple calcifications inside the abscess and extensive destruction of the upper (S1/S2) sacral vertebrae. 


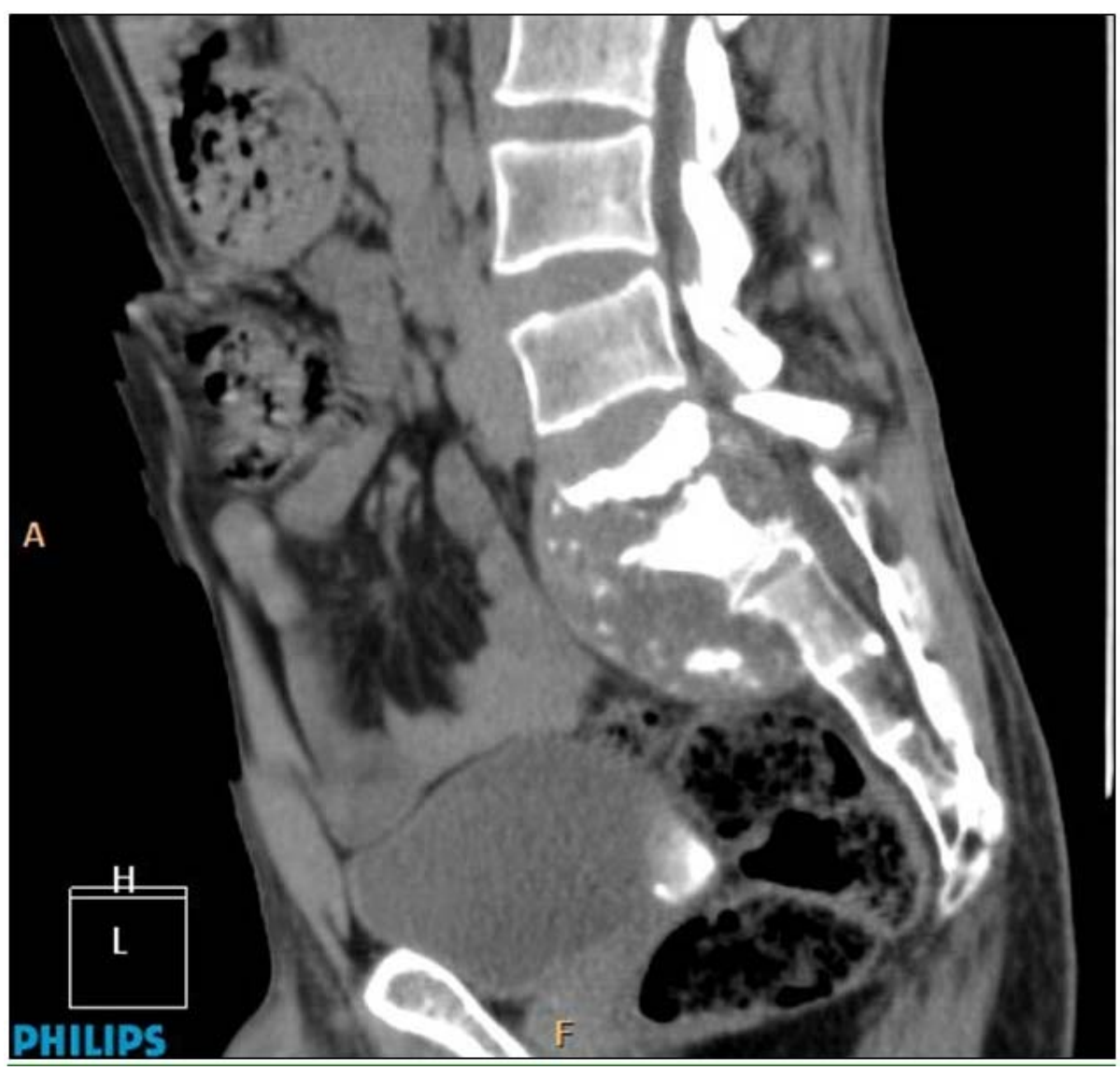

There was destruction of both sacro-iliac joints. This mass fitted the description of an infective process: most likely sacral tuberculosis.

Blood results revealed; normocytic normohromic anaemia, CRP (c-reactive protein) $=64.3$ (normal range $0-10), E S R=3 \mathrm{~mm} / \mathrm{hr}(0-12)$ and the CD4 count of 206. The viral load was below detectable levels. A decision was made to do bone biopsy for a definite diagnosis.

The technique of percutaneous ilio-sacral screw fixation of disrupted sacro-iliac joint is well-described by F. Laude et al [ $\left.{ }^{6}\right]$. We modified this technique and used it for diagnostic biopsy of the S1/S2 sacrum: there is no facility for CT-guided percutaneous biopsy at our institution. What is readily available is C-arm fluoroscopy. The threaded guide-wire is used as in ilio-sacral screw fixation. The appropriate views( lateral, inlet, and outlet) are used to monitor the tract of the guide wire during the wire advancement. The wire is advanced up to the vertebral body of S1. A $6.5 \mathrm{~mm}$ cannulated drill bit is used to make the tract for the core biopsy needle. Thereafter the drill is removed. The Corb ${ }^{\circledR}$ core biopsy needle (small size) ( ZIMMER ${ }^{\circledR}$, SOUTH AFRICA), is advanced over the guide-wire until it reaches the S1 body (fig 2.)

Figure 2: Trans ilio-sacral approach. The lateral view (A) shows placement of threaded guidewire in the S1 vertebral body. The inlet (B) and the outlet (C) views confirm correct placement of the guidewire in the vertebra. Core biopsy needle used to obtain specimens (D). 


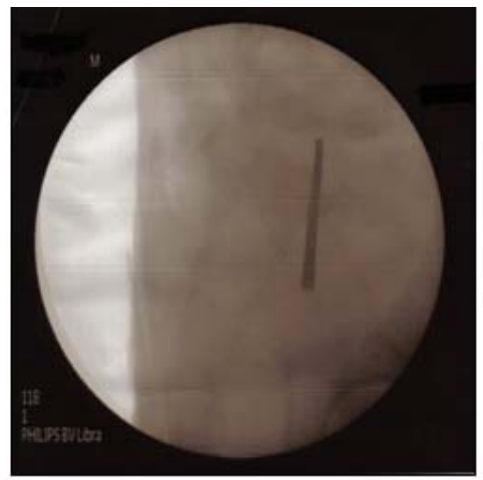

A

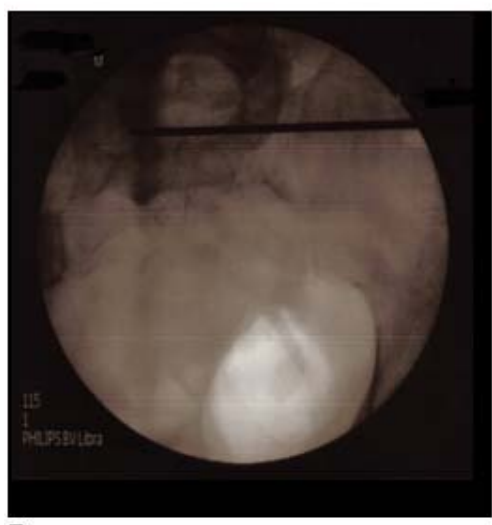

B

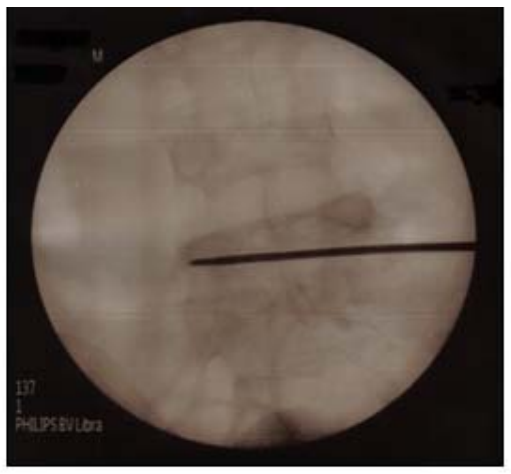

C

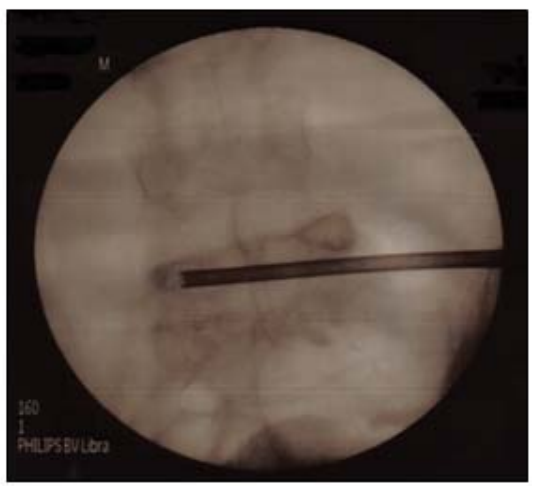

D

The guide-wire is removed. Multiple biopsy specimens are taken. Every time the core biopsy needle is removed, the guide-wire must be re-inserted and its position confirmed with C-arm fluoroscopy. Specimens were sent for acid fast bacilli, microscopy, culture, and sensitivity for bacteria and fungi. After the biopsy, two skin sutures are adequate for closure. We had no complications from this procedure. Histology report stated that there were areas of caseous necrosis and acid-fast bacilli were demonstrated. This clinched the diagnosis of sacral tuberculosis.

\section{Discussion}

The technique we have described offers and alternative to well-described techniques or approaches to the upper sacral lesions. It is fast ( the whole procedure lasted 45 minutes), safe, and very easy to learn and master. It should be considered where facilities for CT- or Ultrasound-guided percutaneous biopsy are not available.

There may be concern about possible contamination of the ipsilateral sacro-iliac joint: the sacro-iliac joint was already involved in this patient. Moreover, sacro-iliac joint tuberculosis is a treatable and curable disease. Sacro-iliac joint tuberculosis has a good outcome[ $\left.{ }^{7}\right]$.

Spinal tuberculosis occurs in $25 \%$ to $60 \%$ of skeletal tuberculosis[ ${ }^{1}$ ]. Spinal tuberculosis is the most severe form of skeletal tuberculosis[ ${ }^{2}$ ]. The lowest part of the vertebral column is rarely involved in spinal tuberculosis. The lumbo-sacral region is involved in less than $5 \%$ of cases $\left[{ }^{2},{ }^{3}\right]$. Isolated sacral tuberculosis is extremely rare. John C. Well et al. $\left[{ }^{8}\right]$ in his extensive literature search in 2004 , found one reported case of sacral tuberculosis.

Atypical spinal tuberculosis, of which sacral tuberculosis is an example, occurs in $2.1 \%$ of spinal infection. $\left[{ }^{9},{ }^{10}\right]$. Atypical spinal tuberculosis must be differentiated from other spinal pathologies like malignancies(5).

Radiological investigations play a crucial role in the pre-operative or diagnostic work-up of patients with spinal pathology. CT-SCAN and magnetic resonance imaging(MRI) are the most informative diagnostic modalities $\left[{ }^{1}\right]$. There are no specific findings diagnostic of spinal or sacral tuberculosis. It is interesting to note that MRI of the sacro-iliac joint in sacral tuberculosis will reveal that up to $10 \%$ of patients with sacral tuberculosis have sacro-iliac joint involvement $\left[{ }^{1}\right]$.

Spinal infection in HIV positive patients can be due to a variety of organisms[ ${ }^{5}$ ]. Spinal biopsy is an important part of patient work-up. Percutaneous CT-guided needle biopsy is a proven diagnostic method of choice [ $\left.{ }^{7}\right]$. It is fast, efficient, cost-effective and has few complications. In experienced hands, this procedure has a complication rate of less than $1 \%$ to $3 \%\left[{ }^{12}\right.$ ]. It has a high diagnostic yield(79.41\%), and a high accuracy rate 
(95.37\%) in deep-seated musculo-skeletal lesions.[ ${ }^{13}$ ].

Overall, percutaneous vertebral body biopsy is positive in $88 \%$ to $100 \%$ of cases. However, positive culture results are not as high: $46 \%$ to $91 \%$.

CT-guided biopsy is the gold standard for pathology of the sacrum. The most straightforward approach is the posterior approach. The serious complication for this procedure is bowel perforation. Pre-sacral and anterior body pathology can be approached via the anterior route. Low-lying lesions can approached via the transrectal route [ ${ }^{12}$ ].

\section{Conclusion}

Percutaneous trans ilio-sacral approach is an alternative safe technique for biopsy of the upper sacral spine.

\section{References}

1. Patankar, T., et al., Imaging in isolated sacral tuberculosis: a review of 15 cases. Skeletal Radiol 2000. 29: p. 392-396.

2. Cabral, M.M.L., et al., Tuberculous spondilytis in teenager. J Bras Pneumol, 2005. 31 (3): p. 261-264.

3. Sakho, Y., et al., Pott's disease in Senegal. Eur J Orthop Surg Traumatol, 2003. 13: p. 13-20.

4. Kumar, A., M.K. Varshney, and V. Trikha, Unusual presentation of Isolated Sacral Tuberculosis. Joint Bone Spine, 2006. 73: p. 751-752.

5. Laude, F. and P. Paillard, Technique of percutaneous transsacral screw stabilization for sacroiliac joint injury and sacral fractures. Maitrise Orthopedique, 2001. 108: p. 1-16.

6. Ramlakan, R.J.S. and S. Govender, Sacroiliac joint tuberculosis. International Orthopaedics (SICOT), 2007. 31: p. 121-124.

7. Wellons, J.C., et al., Sacral Tuberculosis: A case report and review of the literature. Surg Neurol, 2004. 61: p. 136-141.

8. Yalniz, E., G. Pekindil, and S. Aktas, Atypical Tuberculosis of the Spine. Yonsei Med J, 2000. 41(5): p. 657-661.

9. Rahman, N.U., et al., Atipical forms of spinal tuberculosis: a case report and review of the literature. Surg Neurol, 1999. 51: p. 602-607.

10. Bono, C.M., Spectrum of Spine Infections in Patients with HIV. Clinical Orthopaedics and related research, 2006. 444: p. 83-91.

11. Hasegawa, K., et al., Spinal Tuberculosis Report of an Atypical Presentation. Clinical Orthopaedics and Related Research, 2002. 403: p. 100-103.

12. Tehranzadeh, J., C. Tao, and C.A. Browning, Percutaneous Needle Biopsy of the Spine. Acta Radiologica, 2007. 48: p. 860-868.

13. Puri, A., et al., CT-guided percutaneous core needle biopsy in deep seated musculoskeletal lesions: a prospective study of 128 cases. Skeletal Radiol, 2005. 35: p. 138-143.

Home | Journals | Sponsors | Medical Books | PubMed | Contact

Copyright Internet Scientific Publications, LLC, 1996 to 2008. 